\title{
Bispectral index monitoring in spinal anesthesia with sedation can prevent dexmedetomidine related bradycardia: a randomized clinical trial
}

\author{
Jiwook Kim $\bowtie$, Hui Young Kim $\bowtie$, Minsu Yun $\bowtie$, Jeongyeop Lee $\bowtie$, Joo-Duck Kim $\bowtie$, \\ Donghee Kang \\ Author affiliation: \\ Department of Anesthesiology and Pain Medicine, Kosin University College of Medicine, 262 Gamcheon-ro, Seo-gu, Busan \\ 49267, Korea. \\ Correspondence: Donghee Kang; E-mail: lorien@kosin.ac.kr
}

\section{Abstract}

Background: An unintentional large dose of dexmedetomidine during sedation can lead to increased side effects such as bradycardia and hypotension. We investigated whether the frequency of bradycardia in patients undergoing surgery under spinal anesthesia and sedation with dexmedetomidine was effected if bispectral index (BIS) was used to monitor the sedation.

Methodology: Fifty patients between 20 and 60 y of age, who underwent spinal anesthesia, were included in this study. The patients were divided into two groups (control and BIS groups). Patients in the control group were administered a loading dose of $1 \mu \mathrm{g} / \mathrm{kg}$ dexmedetomidine for the first 10 min without BIS monitoring, followed by a maintenance dose of $0.2 \mu \mathrm{g} / \mathrm{kg} / \mathrm{h}$. Patients in the BIS group received a loading dose of $1 \mu \mathrm{g} / \mathrm{kg}$ dexmedetomidine for the first $10 \mathrm{~min}$. The maintenance dose in this group was administered at the discretion of the anesthesiologist to maintain the BIS score between 50 and 70. The baseline heart rate (HR), lowest HR, and the difference between baseline and the lowest HR were calculated.

Results: The baseline HR was $74.4 \pm 11.0$ beats per minute (bpm) and $80.9 \pm 16.0 \mathrm{bpm}$, in the control and BIS groups respectively $(p=0.098)$. The difference between the baseline and lowest HR in the control and BIS groups was 19.4 $\pm 7.6 \mathrm{bpm}$ and $25.5 \pm 8.8 \mathrm{bpm}$, respectively $(p=0.011)$. Low baseline HR was positively correlated with a reduced lowest HR.

Conclusions: The frequency of bradycardia during sedation with dexmedetomidine decreased with BIS monitoring. Patients with a low baseline HR were more likely to develop bradycardia during sedation with dexmedetomidine. Therefore, BIS monitoring may be helpful in patients with low baseline HR.

Key words: Bispectral index monitor; Dexmedetomidine; heart rate; spinal anesthesia

Citation: Kim J, Kim HY, Yun M, Lee J, Kim JD, Kang D. Bispectral index monitoring during sedation with dexmedetomidine in spinal anesthesia prevents bradycardia: a randomized clinical trial. Anaesth. pain intensive care 2021;26(1):14-19 ; DOI: 10.35975/apic.v26i1.1760

\section{Introduction}

Dexmedetomidine is an $\alpha_{2}$-adrenergic receptor agonist with sedative and sympathetic nerve blocking effects that relieve pain and anxiety. It acts on the $\alpha_{2}$-adrenergic receptors in the sympathetic ganglia and can cause both hypertension and hypotension. ${ }^{1}$ Hypertension may occur when dexmedetomidine transiently acts on $\alpha 2 b$ receptors in peripheral vascular smooth muscle. ${ }^{1}$ However, heavy doses of dexmedetomidine may lead to an increased frequency of bradycardia and/or hypotension. ${ }^{2}$ This is due to a vasodilatory effect of dexmedetomidine exerted by acting on central $\alpha 2 \mathrm{a}$ receptors. ${ }^{2}$ Severe bradycardia with a heart rate of $\leq 40$ 


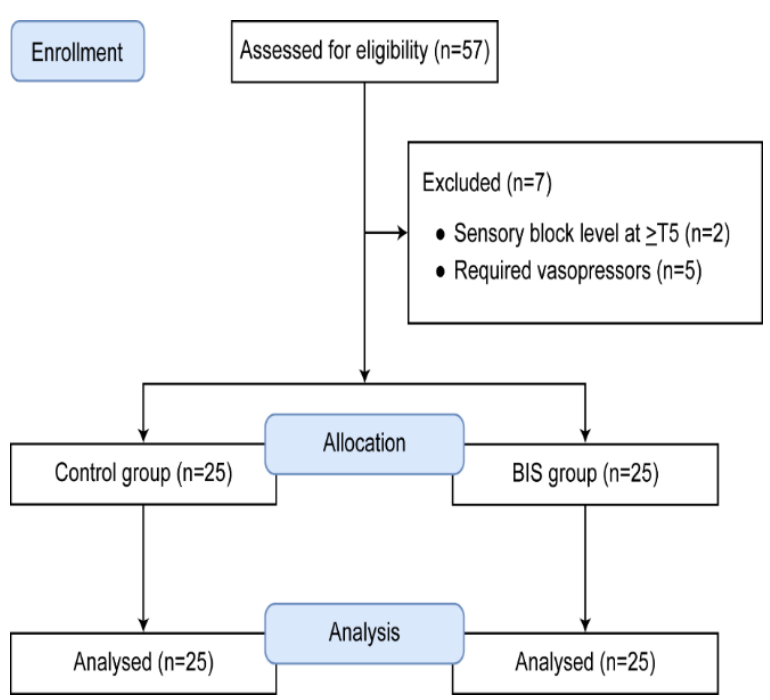

Figure 1: CONSORT flow diagram

beats per minute (bpm) and cardiac arrest have been observed in several patients under sedation with dexmedetomidine. ${ }^{3,4}$ Various regimens have been employed to reduce the bradycardia from dexmedetomidine; such as using dexmedetomidine in combination with other sedatives or administering anticholinergics prophylactically. ${ }^{5,6} \mathrm{We}$ hypothesized that the same effect can be expected if BIS monitoring is used during sedation using dexmedetomidine to optimize the dose of the drug. An earlier study reported that the use of BIS during induction of general anesthesia reduced the amount of anesthetic to be used for the procedure. $^{7}$ The BIS monitors electroencephalographic signals to quantitatively measure the level of patient consciousness using scores ranging from 0 to 100 . It is an effective measure of assessing patient sleep and is highly correlated with the sedation score. ${ }^{8}$ A BIS score of less than 70 indicates deep sedation, and a score of less than 40 indicates deeper sedation with gradual inactivation of the brain. ${ }^{9}$

Bradycardia and hypotension are the known side effects of dexmedetomidine and can be dose dependent. We compared the frequency of these side effects in patients who were monitored using Bispectral array (BIS) compared to controls.

\section{Methodology}

\subsection{Study design and selection criteria}

This prospective, observational study was approved by our institution (vide No. KUGH 2019-05-008-001). All study protocols were compliant with the Declaration of Helsinki of 1975 (revised 2013), and written informed consent was obtained from all patients. This study was conducted on patients, between July 2019 and January
2020. A total of 50 patients aged 20-60 y, American Society of Anesthesiology physical status 1 and 2, were included in the study. They were admitted to our hospital for elective transurethral resection of a bladder tumor under spinal anesthesia in the urology department. Patients who requested sedation during the operation were selected. Patients with cardiovascular problems that could affect the heart rate, those who underwent spinal anesthesia with sensory block level at $\geq \mathrm{T} 5$ such that the cardiovascular system could be affected, and those who required vasopressors after spinal anesthesia were excluded from the study.

\subsection{Anesthesia procedure and BIS monitoring}

Patients were randomly allocated into two groups, using a computer-generated random assignment: a control group and a BIS group. After arriving in the operating room, anesthesia was administered by medical staff unrelated to the study. In both groups, with the patient lying in the lateral decubitus position, $10-12 \mathrm{mg}$ of hyperbaric bupivacaine $0.5 \%$ (Myungmoon Pharmaceutical, Myungmoon, Korea) was injected into the L3-4 interspace or L4-5 interspace. The target level of the spinal sensory block was T10, and the pinprick test was performed to confirm the sensory block.

After confirming spinal anesthesia, dexmedetomidine was administered for sedation.

In the control group, $1 \mu \mathrm{g} / \mathrm{kg}$ dexmedetomidine was administered as the loading dose for the first $10 \mathrm{~min}$, followed by a maintenance dose of $0.2 \mu \mathrm{g} / \mathrm{kg} / \mathrm{h}$ without BIS monitoring (BIS VISTATM, Aspect Medical Systems, Norwood, MA). Similar to the control group, the BIS group was administered a loading dose of 1 $\mu \mathrm{g} / \mathrm{kg}$ dexmedetomidine for the first $10 \mathrm{~min}$. After the loading dose, the maintenance dose of dexmedetomidine was adjusted by an anesthesiologist unrelated to the study starting from $0.2 \mu \mathrm{g} / \mathrm{kg} / \mathrm{h}$ and maintaining the BIS score at 50-70.

Patients' baseline vital signs and BIS scores during stable condition were recorded before administration of spinal anesthesia. Following spinal anesthesia, the vital signs and BIS scores were continuously monitored at 5min intervals. If the heart rate decreased to less than 40 bpm during surgery, $0.2 \mathrm{mg}$ glycopyrrolate was administered intravenously.

\subsection{Statistical analysis}

Statistical analysis was performed using $\mathrm{MedCalc}^{\circledR}$ (version 19.0.3, MedCalc Software, Mariakerke, 


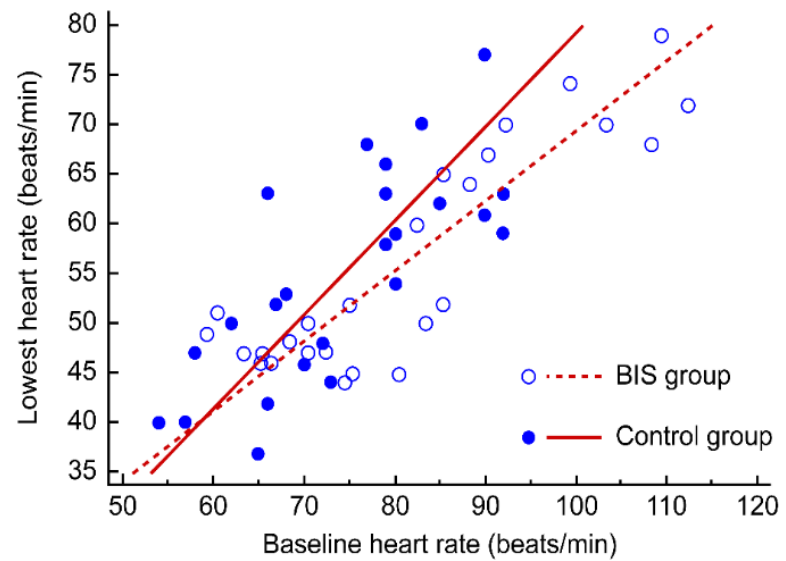

Figure 2: Relationship between baseline heart rate and lowest heart rate

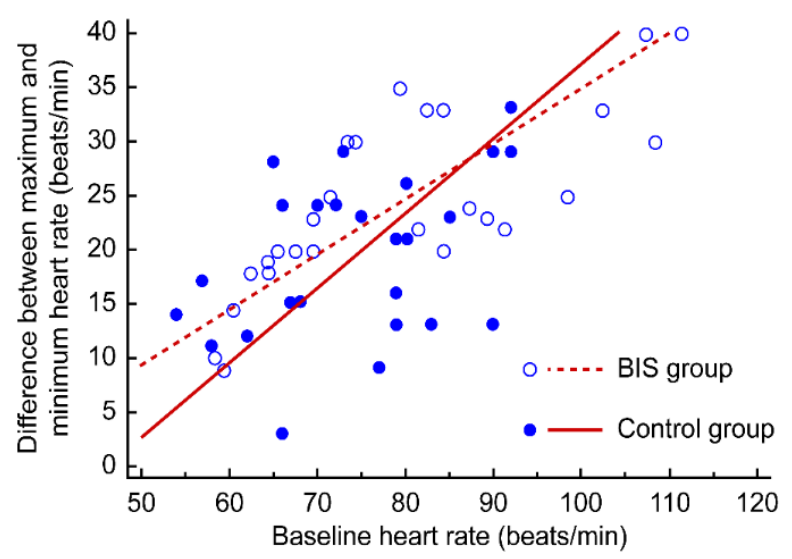

Figure 3: Relationship between baseline heart rate and difference in heart rate (baseline - lowest)

Belgium). Age, height, weight, sensory block level, local anesthetic dose, baseline heart rate, difference between baseline and lowest heart rate (difference in heart rate), loading and maintenance doses of dexmedetomidine, and total dose were compared between groups using independent t-test. Sex and number of glycopyrrolate administrations were compared using the chi-squared test or Fisher's exact test. Pearson correlation analysis was conducted to analyze the correlation between baseline heart rate and difference in heart rate, and between baseline heart rate and the lowest heart rate. A p $<0.05$ was considered significant.

\section{Results}

A total of 50 patients were analyzed in this study (Figure 1). The control and BIS group comprised 25 patients each (control group: 16 males +9 females; BIS group: 12 males +13 females). The results are expressed as mean \pm standard deviation (SD). No significant differences were found in age, height, weight, bupivacaine dose used for spinal anesthesia, or sensory block level between the two groups (Table 1).

\begin{tabular}{|c|c|c|c|}
\hline Variable & $\begin{array}{l}\text { Control } \\
\text { group (n = } \\
25 \text { ) }\end{array}$ & $\begin{array}{l}\text { BIS group } \\
(n=25)\end{array}$ & $\begin{array}{l}\text { P. } \\
\text { value }\end{array}$ \\
\hline \multirow[t]{2}{*}{ Gender } & $16(64.0)$ & $12(48.0)$ & 0.794 \\
\hline & $9(36.0)$ & $13(52.0)$ & \\
\hline Age $(y)$ & $48.4 \pm 11.8$ & $43.3 \pm 10.5$ & 0.115 \\
\hline Height (cm) & $165.2 \pm 8.6$ & $166.0 \pm 9.1$ & 0.775 \\
\hline Weight (kg) & $68.5 \pm 10.5$ & $64.1 \pm 9.7$ & 0.130 \\
\hline $\begin{array}{l}\text { Sensory } \\
\text { block level }\end{array}$ & T9.5 \pm 0.9 & T9.4 \pm 1.2 & 0.795 \\
\hline $\begin{array}{l}\text { Bupivacaine } \\
\text { dose (mg) }\end{array}$ & $10.9 \pm 0.9$ & $10.9 \pm 0.7$ & 1.000 \\
\hline
\end{tabular}

The dexmedetomidine loading doses in the control and BIS groups were $68.5 \pm 10.5$ and $64.1 \pm 9.7 \mu \mathrm{g}$, respectively, which were not significantly different between the two groups $(\mathrm{p}=0.130)$. However, the dexmedetomidine maintenance dose was significantly different between the two groups (control group vs. BIS group; $11.4 \pm 1.7 \mu \mathrm{g}$ vs. $16.8 \pm 6.6 \mu \mathrm{g}$ ). Further, the total dose of dexmedetomidine administered in $1 \mathrm{~h}$ was not significantly different between the two groups (control group vs. BIS group; $79.9 \pm 12.2 \mu \mathrm{g}$ vs. $80.9 \pm 12.2 \mu \mathrm{g}$; $\mathrm{p}=0.770$ ).

The baseline heart rate was $74.4 \pm 11.0 \mathrm{bpm}$ and $80.9 \pm$ $16.0 \mathrm{bpm}$ in the control and BIS group respectively, and the difference between the groups was not significant ( $p$ $=0.098$ ). Further, the lowest heart rate in the control and BIS groups was $55.0 \pm 10.5 \mathrm{bpm}$ and $55.5 \pm 12.2 \mathrm{bpm}$, respectively, and the difference between both groups was not significant $(p=0.872)$. However, the difference between the baseline and lowest heart rate was significantly different between the two groups (control group vs. BIS group; $19.4 \pm 7.6 \mathrm{bpm}$ vs. $25.5 \pm 8.8 \mathrm{bpm}$; $\mathrm{p}=0.011)$ (Table 2).

A positive correlation was observed between the baseline and lowest heart rate $(r=0.735, \mathrm{p}<0.001$, Figure 2) and between the baseline and difference in heart rate $(r=0.641, p<0.001$, Figure 3$)$. Specifically, patients with a low baseline heart rate showed a reduced lowest heart rate, and the difference in heart rate was 


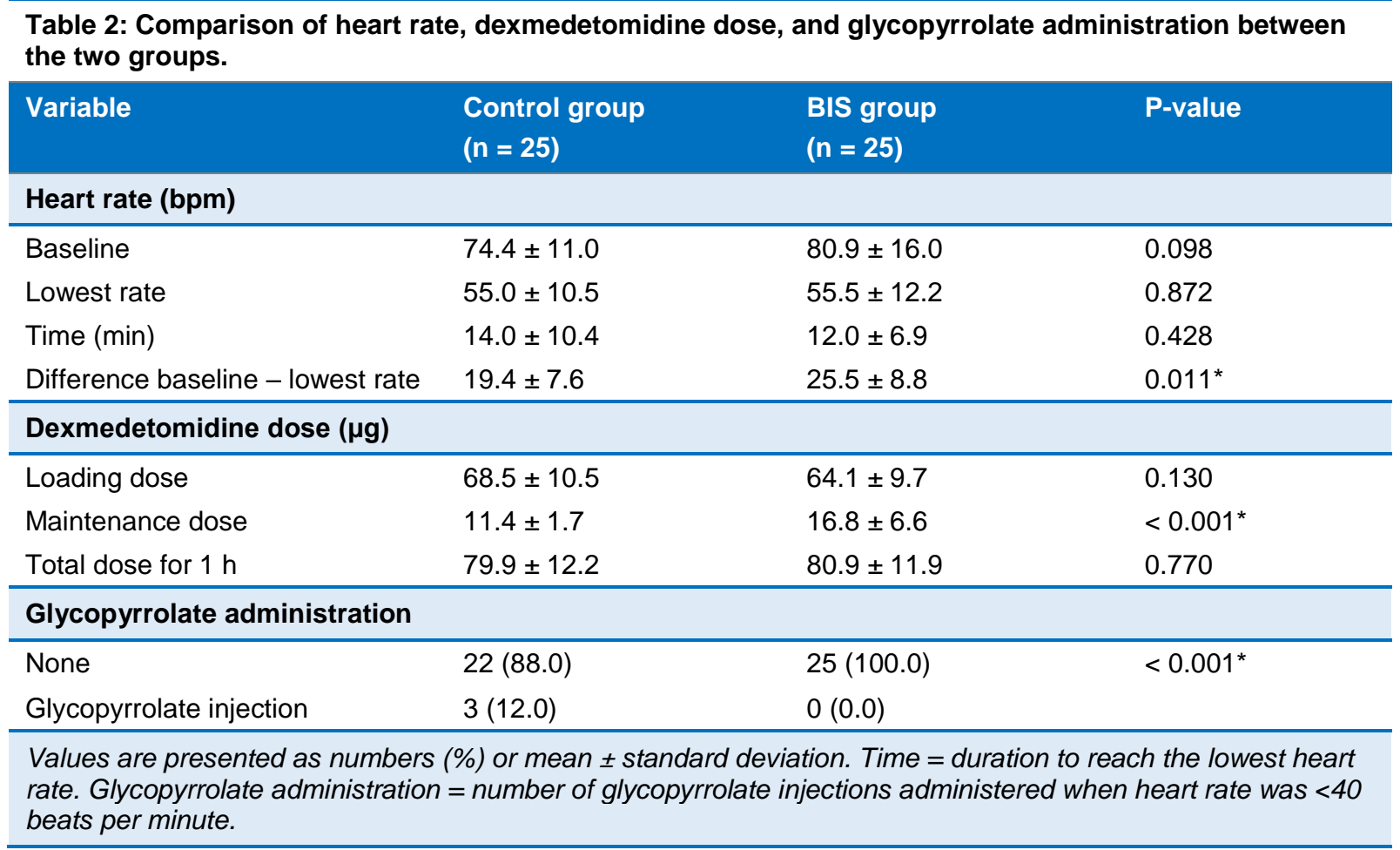

greater in patients with a high baseline heart rate.

Three patients in the control group were administered $0.2 \mathrm{mg}$ glycopyrrolate during surgery due to a decrease in the heart rate to less than $40 \mathrm{bpm}$; administration of glycopyrrolate was not required in the BIS group.

\section{Discussion}

Dexmedetomidine is widely used for sedation during spinal anesthesia. As an $\alpha_{2}$-adrenergic receptor agonist, it has sedative, analgesic, and sympathetic nerve inhibiting effects. Dexmedetomidine has a 1600 times greater binding affinity for $\alpha_{2}$ receptors than for $\alpha_{1}$ receptors, ${ }^{10}$ and it is widely used for sedation owing to its minimal effects on respiratory suppression, compared with other sedatives such as propofol or opioids. ${ }^{11}$ Furthermore, dexmedetomidine affects the cardiovascular system, which leads to biphasic blood pressure responses, causing the blood pressure to temporarily increase and gradually decrease afterward. As blood pressure increases, the heart rate decreases due to a decreased sympathetic tone and increased vagal tone. ${ }^{12,13}$

Such a decrease in heart rate is commonly observed during sedation using dexmedetomidine, and in some patients, severe bradycardia and cardiac arrest with a heart rate of $\leq 40 \mathrm{bpm}$ have been observed. ${ }^{3,4}$ The frequency of bradycardia caused by dexmedetomidine administration can be reduced by preventing excessive use of dexmedetomidine and unnecessary deep sedation.
Among methods including Observer's Assessment of Awareness/Sedation Scores, Self-Sedation Scores, and BIS monitoring during sedation, BIS monitoring was chosen and used to prevent excessive sedation. ${ }^{14}$

The dexmedetomidine loading dose administered in both groups of this study was $1 \mu \mathrm{g} / \mathrm{kg}$, corresponding to previous findings that $1 \mu \mathrm{g} / \mathrm{kg}$ dexmedetomidine helps achieve quicker sedation without serious complications, compared with $0.5 \mu \mathrm{g} / \mathrm{kg}$ loading dose. ${ }^{15}$ Furthermore, the maintenance dose in the control group was set as 0.2 $\mu \mathrm{g} / \mathrm{kg} / \mathrm{h}$, as it has been previously demonstrated that compared with $0.4 \mu \mathrm{g} / \mathrm{kg} / \mathrm{h}, 0.2 \mu \mathrm{g} / \mathrm{kg} / \mathrm{h}$ is the optimal dose to maintain a BIS score of 60-80, with less effects on heart rate and blood pressure. ${ }^{16}$ Additionally, the maintenance dose did not exceed $0.5 \mu \mathrm{g} / \mathrm{kg} / \mathrm{h}$ in the BIS group, as a previous study has demonstrated that excessive sedation and airway obstruction may occur with a loading dose of $1 \mu \mathrm{g} / \mathrm{kg}$ and maintenance dose of $0.5 \mu \mathrm{g} / \mathrm{kg} / \mathrm{h}^{17}$

The dexmedetomidine maintenance dose was significantly different between the two groups $(\mathrm{P}<0.001)$. We expected that BIS monitoring would prevent unnecessary administration of dexmedetomidine and thereby excessive sedation, leading to a decreased total volume of administered dexmedetomidine. However, the maintenance dose was higher in the BIS group than in the control group. In previous studies, the BIS has been set between 55 and 80 for shallow sleep. ${ }^{9}$ By contrast, we set BIS between 
50 and 70 to control the maintenance dose, which might have led to an higher maintenance dose in the BIS group compared to the control group. ${ }^{9}$ Interestingly, although the maintenance dose was greater in the BIS group, bradycardia with a heart rate of $<40 \mathrm{bpm}$ was observed in only one patient in the BIS group, compared to three patients in the control group ( $\mathrm{p}<0.001)$. The fixed maintenance dose used in the control group might have been too excessive for adequate sedation in some patients, leading to more cases of bradycardia. By contrast, BIS monitoring in the BIS group allowed flexible adjustment of the dexmedetomidine maintenance dose, which might have contributed to the reduced number of cases with bradycardia.

The difference in heart rate was also significantly different between the two groups $(p=0.011)$. The difference between the baseline and lowest heart rate can be affected by a wide range of factors, and an increase in the difference in heart rate is directly proportional to the increased dose of dexmedetomidine used. ${ }^{2}$ However, the total dose of dexmedetomidine used during the one hour of the surgery was not significantly different between the two groups. Therefore, the total dose of dexmedetomidine did not seem to have a significant effect on the difference in heart rate in this study. Another factor that affects the difference in heart rate may be the baseline heart rate. We observed that patients with a higher baseline heart rate had a greater decrease in heart rate during sedation using dexmedetomidine (Figure 3). Additionally, a low baseline heart rate was positively correlated with a reduced lowest heart rate. Therefore, patients with a low baseline heart rate may have an increased chance of developing bradycardia that requires treatment (Figure 2). Overall, BIS monitoring might help control the dexmedetomidine dose and reduce the possibility of bradycardia in patients with a low baseline heart rate.

\section{Limitations}

One limitation of this study was that the dexmedetomidine loading dose was determined based on the body weight of the patients in both groups, and BIS monitoring was used to control only the maintenance dose. However, controlling the loading dose through BIS monitoring may also lead to different results regarding the use of dexmedetomidine. Additionally, in a previous study, bradycardia was reported in $13 \%$ of the patients, when the spinal sensory block level was higher than T5. ${ }^{18}$ In this study, although the sensory block level did not exceed T8 and differed between the two groups, the sensory block level might have still affected the heart rate.

\section{Conclusion}

We expected that monitoring BIS during sedation with dexmedetomidine could reduce the dose used and reduce side effects. As a result of the study, the use of BIS monitoring could not reduce the total drug use. However, it can be seen that in the sedation in which the dexmedetomidine maintenance dose was adjusted under BIS monitoring, bradycardia occurred less than when the maintenance dose was $0.2 \mu \mathrm{g} / \mathrm{kg} / \mathrm{hr}$ without BIS monitoring. The baseline and lowest heart rates were positively correlated, suggesting that the possibility of bradycardia was higher in patients with a low baseline heart rate while using dexmedetomidine for sedation. Therefore, BIS monitoring in patients with a low baseline heart rate during sedation with dexmedetomidine during spinal anesthesia might help prevent bradycardia that requires treatment.

\section{Conflict of interest}

None declared by the authors

\section{Authors' contribution}

JK: Writing; review \& editing

HK: Writing; original draft

MY, JL: Data curation

JK: Conceptualization

DK: Project administration; Writing; review \& editing; Supervision

All authors of this paper have read the manuscript and approved it for submission.

\section{References}

1. Pandharipande $P$, Ely EW, Maze M. Dexmedetomidine for sedation and perioperative management of critically ill patients. Seminars Anesth Periop Med Pain. 2006;25:43-50. DOI: 10.1053/j.sane.2006.01.001

2. Ebert TJ, Hall JE, Barney JA, Uhrich TD, Colinco MD. The effects of increasing plasma concentrations of dexmedetomidine in humans. Anesthesiology. 2000;93:382394. [PubMed] DOI: $10.1097 / 00000542-200008000-00016$

3. Ingersoll-Weng E, Manecke Jr GR, Thistlethwaite PA. Dexmedetomidine and cardiac arrest. Anesthesiology. 2004;100:738-739. [PubMed] DOI: 10.1097/00000542200403000-00040

4. Bharati S, Pal A, Biswas C, Biswas R. Incidence of cardiac arrest increases with the indiscriminate use of dexmedetomidine: a case series and review of published case reports. Acta Anaesthesiol Taiwan. 2011;49:165-167. [PubMed] DOI: 10.1016/j.aat.2011.11.010

5. Park SW, Choi JH, Kim HT, Cho YH. Replacement of dexmedetomidine loading with midazolam for sedation in elderly patients with spinal anesthesia. Medicine (Baltimore). 2018;97(39):e12565. [PubMed] DOI: 10.1097/MD.0000000000012565

6. Ahn EJ, Park JH, Kim HJ, Kim KW, Choi HR, Bang SR. Anticholinergic premedication to prevent bradycardia in combined spinal anesthesia and dexmedetomidine sedation: a randomized, double-blind, placebo-controlled study. J Clin 
Anesth. 2016:35:13-19. [PubMed] DOI 10.1016/j.jclinane.2016.07.012

7. Gürses E, Sungurtekin H, Tomatir E, Dogan H. Assessing propofol induction of anesthesia dose using bispectral index analysis. Anesth Analg. 2004:98:128-131. [PubMed] DOI: 10.1213/01.ANE.0000090314.43496.1D

8. Park EJ, Kim HJ, Park CJ, Yum KW. Relationship between bispectral index, sedation score and plasma concentration, during midazolam sedation (in English with abstract in Korean). J Korean Dent Soc Anesthesiol. 2001;1:21-25. DOI: 10.17245/jkdsa.2001.1.1.21

9. Giménez S, Romero S, Alonso JF, Mañanas MÁ, Pujol A, Baxarias $\mathrm{P}$, et al. Monitoring sleep depth: analysis of bispectral index (BIS) based on polysomnographic recordings and sleep deprivation. J Clin Monit Comput. 2017;31:103-110. [PubMed] DOI: $10.1007 /$ s 10877-015-9805-5

10. Hayashi $Y$, Maze M. Alpha 2 adrenoceptor agonists and anaesthesia. Br J Anaesth. 1993;71:108-118. [PubMed] DOI: 10.1093/bja/71.1.108

11. Venn RM, Hell J, Grounds RM. Respiratory effects of dexmedetomidine in the surgical patient requiring intensive care. Crit Care. 2000;4:302-308. [PubMed] DOI: $10.1186 / c c 712$

12. Bloor BC, Ward DS, Belleville JP, Maze M. Effects of intravenous dexmedetomidine in humans. II. Hemodynamic changes. Anesthesiology. 1992;77:1134-1142. [PubMed] DOI: 10.1097/00000542-199212000-00014

13. Hall JE, Uhrich TD, Barney JA, Arain SR, Ebert TJ. Sedative, amnestic, and analgesic properties of small-dose dexmedetomidine infusions. Anesth Analg. 2000;90:699-705. [PubMed] DOI: 10.1097/00000539-200003000-00035

14. Pollock JE, Neal JM, Liu SS, Burkhead D, Polissar N. Sedation during spinal anesthesia. Anesthesiology. 2000;93:728-734. [PubMed] DOI: 10.1097/00000542-200009000-00022

15. Sim JH, Yu HJ, Kim ST. The effects of different loading doses of dexmedetomidine on sedation. Korean $\mathrm{J}$ Anesthesiol. 2014;67:8-12. [PubMed] DOI: 10.4097/kjae.2014.67.1.8

16. Ok HG, Baek SH, Baik SW, Kim HK, Shin SW, Kim KH. Optimal dose of dexmedetomidine for sedation during spinal anesthesia. Korean J Anesthesiol. 2013;64:426-431. [PubMed] DOI: $10.4097 / \mathrm{kjae} .2013 .64 .5 .426$

17. Yeom JH, Oh MK, Ahn DW, Park SI. A loading dose of $1 \mu \mathrm{g} / \mathrm{kg}$ and maintenance dose of $0.5 \mu \mathrm{g} / \mathrm{kg} / \mathrm{h}$ of dexmedetomidine for sedation under spinal anesthesia may induce excessive sedation and airway obstruction. Anesth Pain Med. 2016;11(3):255-259. DOI: 10.17085/apm.2016.11.3.255

18. Carpenter RL, Caplan RA, Brown DL, Stephenson C, Wu R. Incidence and risk factors for side effects of spinal anesthesia. Anesthesiology. 1992;76:906-916. [PubMed] DOI 10.1097/00000542-199206000-00006 